Research Article

\title{
Application and Effect of the Gymnastic Exercise Mode during Chemotherapy for Breast Cancer Patients
}

\author{
Feifei Xie \\ Department of Humanities and Social Sciences, Zhejiang Industry Polytechnic College, Shaoxing 312000, Zhejiang, China \\ Correspondence should be addressed to Feifei Xie; 20010013@zjipc.edu.cn
}

Received 7 August 2020; Revised 2 September 2020; Accepted 11 September 2020; Published 21 September 2020

Academic Editor: Tifeng Jiao

Copyright (c) 2020 Feifei Xie. This is an open access article distributed under the Creative Commons Attribution License, which permits unrestricted use, distribution, and reproduction in any medium, provided the original work is properly cited.

\begin{abstract}
Breast cancer is one of the most common malignant tumors in women, which seriously threatens the health of women. With the improvement of living standards, the incidence rate of breast cancer is also rising. In the past ten years, the incidence rate of breast cancer in China's major cities has increased by 37\%, far higher than that in Europe and America. At present, chemotherapy and radiotherapy are the main treatment methods for breast cancer, but many patients will have cancer-related fatigue after surgery. Some studies believe that appropriate sports can improve cancer-related fatigue, but there is no specific research in this area. In view of this problem, this paper puts forward a rehabilitation training method based on gymnastics for breast cancer surgery. This paper is divided into three parts. The first part is the basic theory and core concept of breast cancer and cancer-related fatigue. Through the in-depth study of the theory, this paper believes that breast cancer patients paying attention to rehabilitation training can effectively improve cancer-related fatigue and affect the final therapeutic effect. The second part is the rehabilitation training program based on the way of gymnastics. The corresponding experimental model is established by using real cases as samples. In order to ensure the quality of the experiment, this paper gives the treatment plan in detail and establishes a unified evaluation system. In the third part of this paper, the relevant experiments and results analysis are given, and through data analysis, this paper believes that gymnastics can effectively help breast cancer patients with postoperative rehabilitation and continuous recovery of the upper limb function and improve cancer-related fatigue and other issues.
\end{abstract}

\section{Introduction}

Breast cancer is one of the most common malignant tumors in women. According to statistics, more than 1.32 million women suffer from breast cancer every year, and 560000 women die of breast cancer. The incidence rate of breast cancer is the highest in developed countries in North America, Western Europe, and Northern Europe and the lowest in Africa. The incidence rate of breast cancer is increasing all over the world. The incidence rate of breast cancer is increasing at $6 \%$ to $25 \%$ in high incidence areas and low incidence areas. With the improvement of living standards in Asian countries, the incidence rate of breast cancer in Asian women is significantly higher than that in Europe and America and has become one of the most popular areas of breast cancer incidence rate in Asia. China's incidence rate of breast cancer has increased by $41 \%$ in the past ten years. City cancer experts have shown that breast cancer has become the leading cause of cancer in some cities, especially in Shanghai, Beijing, and coastal areas. It is the highest incidence area of breast cancer in China, and the highest in Shanghai.

At present, the treatment of breast cancer mainly adopts the comprehensive treatment mode of surgery combined with radiotherapy, chemotherapy, endocrine therapy, immunobiological therapy, and various supportive therapies. Among them, chemotherapy has become an important treatment for breast cancer. With the deepening of the understanding of cancer and the continuous breakthrough of treatment technology, new chemotherapy methods are constantly added in cancer chemotherapy. The basic purpose of chemotherapy is to reduce the local lesions, surrounding tissue infiltration and lymph node metastasis, improve the surgical resection rate or increase the curative effect of 
radiotherapy, eliminate micrometastasis, or delay the process of tumor metastasis. With the improvement of diagnosis and comprehensive treatment technology, the survival time of breast cancer patients has been prolonged. According to recent estimates, in the United States, 99\% of breast cancer patients without metastatic disease and $85 \%$ of patients with local metastasis survive for more than five years. Chinese scholars followed up breast cancer patients diagnosed in a cancer hospital from 1975 to 2000. They found that the 3-year survival rate of breast cancer was $83.2 \%$ in the 1980 s and $84.9 \%$ in the 1990 s.

In different stages of treatment, cancer patients need to receive different intensity and methods of chemotherapy and radiation therapy, which can kill tumor cells and normal tissue cells and lead to different degrees of toxicity and side effects, complications and even be life-threatening. Severe side effects include gastrointestinal reactions such as nausea and vomiting, bone marrow suppression, leukocyte predominance, decreased hemoglobin, and neurotoxicity. It is characterized by numbness and pain of limbs, as well as other liver and kidney damage and cardiac toxicity. Some scholars have found that many chemotherapeutic drugs, such as platinum, tubulin inhibitors, borosamine, and sallidol, are widely used in the treatment of solid tumors and hematological malignancies. Many chemotherapy drugs, such as platinum, microtubulin inhibitors, boromine, and thalidol, have neurotoxicity, causing a series of nerve disorders in peripheral nerve or autonomic nerve injury, known as peripheral neuropathy caused by chemotherapy. There are also views that breast cancer surgery, radiotherapy, and chemotherapy patients in pain and tension for a long period of time affect the patient's physical and mental health and can make the patient appear weak and have other symptoms, such as very low mood, the whole body decline, drowsiness, fatigue, and interest decline, such as in cancer, because it is tiring. The best way to improve cancer-related fatigue is exercise therapy, such as core strength training and lower limb strength training, and postoperative rehabilitation exercise training research on breast cancer is less. At present, there is a unified guidance in this field, and a solution based on gymnastics in chemotherapy for breast cancer patients is proposed.

First of all, the core theory of breast cancer and cancerrelated fatigue was deeply studied, and it was concluded that breast cancer chemotherapy had a certain impact on women's body structure, including physiological and psychological effects. It is for this reason that many women are emotional and physically tired after surgery. At present, the treatment of breast cancer has been relatively complete and greatly improved the survival rate of patients, but little attention has been paid to the postoperative rehabilitation training, which is also one of the important problems to be solved in this field. The rehabilitation training method based on gymnastics is specially customized according to the postoperative needs of breast cancer patients. It can strengthen the training of upper limb muscle strength and reduce the training burden of patients. This paper presents a rehabilitation method for breast cancer patients based on gymnastics and establishes the corresponding experimental model. The experimental samples in this paper are all from real cases, after screening, and they are divided into an intervention group and control group. The intervention group uses gymnastics training for exercise intervention, while the control group uses individual free training. At the end of the article, through a number of comparative experiments including upper limb dysfunction and uncertainty of disease, through the analysis of experimental data, it is believed that the gymnastics training method in this paper can help patients recover upper limb function quickly after chemotherapy and improve their mental health. This experiment has achieved a better effect and further verified that gymnastics can play a positive role in the postoperative rehabilitation of breast cancer patients [1-3].

\section{Basic Theories and Core Concepts of Breast Cancer}

2.1. Epidemiology of Breast Cancer. Breast cancer is a serious threat to women's health in the world. It is also one of the common malignant tumors of women. There are about 1.32 million women suffering from breast cancer every year in the world, and 560000 women die of breast cancer. On average, one woman is diagnosed with breast cancer every 25 seconds and one woman loses her life every 80 seconds. In Canada and other European and American countries, breast cancer is the leading cause of female cancer. One in seven women has breast cancer, which is one of the main causes of female death. Our country originally belonged to a low incidence rate of breast cancer. But, in the recent years, with the development of social economy and the improvement of people's living standard, the incidence rate of breast cancer has also been increasing. In China, in Beijing, Guangzhou, Shanghai, and Shenzhen, the incidence rate of breast cancer has ranked the city's leading place in women's malignant tumors, rising from 16/10 million in 1995 to $56 / 10$ million in 2005 . Compared with foreign countries, the incidence rate of breast cancer in Europe and the United States is $1 / 10$, although China is still at a low level, but the growth rate is too fast. It is still growing at $3 \%$ to $4 \%$ per year, and its growth rate is faster than that of Europe and America. In addition, the incidence rate of breast cancer is obviously in younger people in the recent years, especially for the patients aged 22 33 years. There is another $42 \sim 50$ years of age after the peak. This is 10 years ago as a whole in the western countries with a peak age of $40 \sim 50$ years than the female breast cancer. The incidence rate is high, but most of them are elderly women. The incidence of female diseases in China is positive. Especially for the society and families, their morbidity is disastrous. The incidence rate of breast cancer in China still has some outstanding characteristics. The city is higher than in the rural areas, and the knowledge level of the developed areas is higher than that of the less developed areas, the income level is lower than that of the less developed ones, and the income is higher than the lower income $[4,5]$. 
2.2. History and the Current Status of Breast Cancer Treatment. The treatment of breast cancer mostly adopts comprehensive treatment methods, including surgical treatment, radiotherapy, chemotherapy, biological therapy, and endocrine therapy. In the past 20 years, with the deepening understanding of the pathogenesis and biological characteristics of breast cancer and the improvement of early diagnosis and adjuvant treatment technology, systematic comprehensive treatment of breast cancer has been paid attention to and developed. In developed countries, breast conserving surgery for early breast cancer has been recognized and gradually replaced radical or modified radical mastectomy. The literature shows that the curative effect of radiotherapy and chemotherapy for breast cancer with breast augmentation stage I and stage II is not lower than that of radical breast cancer.

At the end of the 19th century, radical mastectomy was established based on anatomy. This method mainly focused on the prevention and treatment of local lymphatic spread of tumor cells. However, a large number of clinical data show that radical mastectomy has poor efficacy. In the 1920s, the reason why radical mastectomy was not designed based on radical mastectomy was that radical mastectomy failed. However, the clinical results showed that the curative effect of expansion radical operation was not better than that of radical operation. Soon, we found that the thoracic fascia can prevent the invasion of cancer cells. The modified radical operation of thoracic fascia with preservation of pectoralis major muscle can not only preserve the main function of the upper limb and good appearance of the chest but also achieve the effect of radical operation. It was not until 1980s that people proposed that distant metastasis of breast cancer preceded lymph node metastasis and that breast cancer was not a local cancer, but a systemic disease. Studies have shown that the metastasis of breast cancer cells is disorderly jumping, rather than from near to far, which provides a theoretical basis for the comprehensive treatment of breast cancer, reducing the scope of surgery and breast conserving surgery. A large number of clinical studies in developed countries have shown that breast conserving surgery combined with radiotherapy is as effective as mastectomy. In the recent years, efforts have been made to develop new treatment strategies to reduce tumor-related symptoms, maintain the quality of life of patients, and maximize the survival rate of breast cancer patients. Rehabilitation training has been widely used in clinical practice because of its advantages in relieving fatigue symptoms, enhancing the physiological function of patients, and regulating the psychology of patients. The general trend of surgical treatment for breast cancer is that the scope of surgery tends to be reasonable and multidisciplinary and systematic treatment should be emphasized [6-8].

2.3. Concept of CRF. Fatigue is a kind of clinical symptom that is difficult to define. In a broad sense, fatigue is a subjective feeling of weakness and lack of passion. Many physiological and psychological diseases have fatigue manifestations. Fatigue is one of the common feelings of normal people. Although it is often associated with systemic objective consumption, its self-evaluation can only be reported by the patient subjectively and cannot be directly tested by the observer, so it is difficult to conduct clinical research. Cancer-induced fatigue (CRF) is one of the most important symptoms of cancer patients. It is a common symptom of cancer patients and cancer patients. It may be caused by cancer itself, or it may be the result of cancer treatment, such as chemotherapy, radiotherapy, and biological therapy.

Fatigue is defined as a subjective, unpleasant symptom, ranging from fatigue to a feeling of exhaustion, as well as systemic conditions that may interfere with a person's daily life. The United States Cancer Network (NCCN) defines CRF as a persistent and universal subjective fatigue feeling, which is related to cancer itself and cancer treatment that affects physiological function. This definition highlights the characteristics of CRF in order to distinguish it from the fatigue experienced by healthy people, which can be recovered by adequate rest and sleep. CRF is a subjective perception of unusual systemic fatigue, related to cancer and cancer treatment, affected by the physiological cycle, the duration and intensity are different, and its remission is disproportionate or unrelated to the individual's behavior and effort. For cancer patients, cancer-related fatigue often becomes chronic, leading to unpleasant feelings. Cancer patients describe fatigue as, lack of vitality, inability to concentrate, weakness, lethargy, and depression. In view of the importance of CRF, the 10th edition of the international classification of diseases (ICD) lists CRF as an independent diagnosis and describes $\mathrm{CRF}$ as nonspecific fatigue, weakness, systemic decay, and drowsiness. At present, cancerrelated fatigue is usually described as a series of subjective feelings caused by chronic stress and pain caused by cancer and its related treatment, such as weakness, activity intolerance, inattention, and decreased motivation or interest. It seriously affects patients' work, study, entertainment, housework, and family life and seriously affects patients' selfcare ability and quality of life [9-11].

2.4. Intervention Effect of Exercise on CRF. For a long time, the cytotoxicity of cancer and cancer treatment, coupled with physical consumption, is easy to lead to fatigue, resulting in increased physical consumption in daily activities. Over the years, doctors have often advised cancer patients to rest and reduce activity, based on their experience, because cancer and related treatments can lead to reduced levels of physical activity, while exercise can cause symptoms such as fatigue, dyspnea, and tachycardia. Avoidance can reduce discomfort; however, in order to avoid the opposite effect of exercise recommendations, reducing activity leads to muscle loss and decreased cardiopulmonary function, which only increases fatigue and other side effects, which may explain the function of feeling tired and physically damaged after treatment. In the recent years, scientific evidence has changed the relationship between exercise, rest, and fatigue. It seems counterintuitive that learning exercise can relieve fatigue during or after 
treatment. But, muscle and blood volume have adaptive changes, increased lung ventilation and perfusion, enhanced heart reserve, and highly concentrated muscle oxidase, so exercise can relieve fatigue by normalizing body function.

Exercise training, even moderate exercise, can make the heart and lung produce more adaptive response, increase cardiac output, reduce heart rate, reduce fatigue, and reduce the need for physical strength. Another hypothesis is that exercise training can accelerate circulation, reduce some cytotoxins or other substances, and reduce the fatigue response. In a study on the relationship between exercise training, fatigue, and quality of life in patients with breast cancer chemotherapy, some scholars found that 7 weeks of family aerobic exercise can improve the quality of life by reducing fatigue. In addition, exercise can also improve mood, improve immunity, reduce vomiting and other side effects, reduce depression, improve sleep, improve disease adaptability and self-efficacy, and ultimately improve the quality of life. Therefore, exercise directly or indirectly reduces the degree of fatigue and improves the quality of life $[12,13]$.

\section{Rehabilitation of Breast Cancer Patients after Operation Based on Gymnastics}

3.1. Research Object and Grouping. The subjects were women with breast cancer who were admitted to the General Service Department of a hospital from May 2019 to October 2019. Among the 120 patients who met the inclusion criteria, 10 of them did not receive chemotherapy during the operation or dropped out of chemotherapy, and did not fall off because of death or serious complications. A total of 110 patients completed the experiment, with an average age of $47.36 \pm 8.26$ years. One hundred and ten people were divided into two groups, with 55 people in each group.

\subsection{Inclusion and Exclusion Criteria. Inclusion criteria:}

(1) The patient was $18-70$ years old. The condition was stable after chemotherapy. The first cycle of chemotherapy had ended, and the second cycle of chemotherapy was in the outpatient department.

(2) There is no regular exercise habit after breast cancer surgery. In this study, regular moderate intensity gymnastics should be no more than four times a week, no more than 80 minutes each.

(3) There are no serious health problems, and the body is suitable for 7 weeks of gymnastics intervention.

(4) The revised Piper Fatigue Scale showed cancer-related fatigue symptoms.

(5) Those who voluntarily participated in the study and completed the informed consent form.

Exclusion criteria:

(1) The main health problems that may affect exercise intervention include coronary heart disease, acute and chronic respiratory diseases, severe hypertension, diabetes, thyroid disease, tuberculosis, anemia, breast cancer, malignant tumor, severe liver and kidney dysfunction, diseases affecting bone and joint movement, and mental disorders

(2) Distant metastasis or recurrence occurred

(3) Regular exercise habits after breast cancer surgery; in this study, regular moderate intensity gymnastics exercise more than four times a week, lasting more than 80 minutes

3.3. Materials and Instruments. In this experiment, 23 kinds of materials and instruments including different dyes were used. The specific name, model, and manufacturer are shown in Table 1.

3.4. Peripheral Blood Sample Collection. After the patient signed the informed consent, $10 \mathrm{ml}$ of superficial venous blood was collected by using an EDTA anticoagulant vacuum container before the first chemotherapy. After the completion of the first chemotherapy, the pollution of $3 \mathrm{ml}$ blood was extracted and discarded for the first time to eliminate the skin epithelial tissue. After the blood was collected, it was immediately reversed and gently mixed to avoid coagulation. According to the Iset standard test procedure, CTC was detected within 1.5 hours. When ctcbiopsy is used within 1.5 hours, the biopsy should be placed at $5^{\circ} \mathrm{C}$ and the blood samples should be kept for no more than 24 hours. The blood samples to be transported should be placed vertically in the specimen transport box with low temperature $\left(5^{\circ} \mathrm{C}-10^{\circ} \mathrm{C}\right)$ to prevent reversion and violent shaking.

\subsection{Chemotherapeutic Drugs and Drug Regimen}

(1) Xiaoyangping injection: the component of this product is rattan, and the auxiliary material is polysorbic acid vinegar.

(2) Chemotherapy drug: docetaxel $65 \mathrm{mg} / \mathrm{m}^{2}$; adriamycin $45 \mathrm{mg} / \mathrm{m}^{2}$.

(3) Chemotherapy group and combination group: chemotherapy group, chemotherapy regimen: docetaxel $63 \mathrm{mg} / \mathrm{m}^{2}$; adriamycin $45 \mathrm{mg} / \mathrm{m}^{2}$. On the first day, 23 repetitions and 7 cycles were performed. The combination group was given xiaoxinping injection on the basis of chemotherapy, once a day, intravenous administration of $8 \%$ glucose injection $280 \mathrm{ml}+60 \mathrm{ml}$ xiaoxinping injection, starting from the first day of chemotherapy, and xiaoxinping injection was given intravenously for 15 consecutive days.

\subsection{Observation Index}

(1) Clinical symptoms: nausea and vomiting, alopecia, diarrhea, and other side effects of chemotherapy

(2) Laboratory indicators: bone marrow suppression and liver function damage

(3) ECG changes 
TABLE 1: List of experimental materials and instruments.

\begin{tabular}{|c|c|c|}
\hline \multicolumn{3}{|c|}{ Blood samples } \\
\hline Peripheral blood $5 \mathrm{ml}$ in patients with breast cancer & & On-site \\
\hline \multicolumn{3}{|c|}{ Reagent } \\
\hline $0.9 \%$ normal saline & & Self-control \\
\hline Neutral balsam & & Zhuhai Besso \\
\hline Medical alcohol & & Domestic \\
\hline Superclean sealed tablets & & Zhuhai Besso \\
\hline Deionized water (DI) & & Self-control \\
\hline Diff staining solution & & Zhuhai Besso \\
\hline Polyoxymethylene solution (PFA) & & EMS \\
\hline PBS buffer & & Domestic \\
\hline \multicolumn{3}{|c|}{ Consumables } \\
\hline Cell filter & & Wuhan Youzhiyou company \\
\hline EDTA vacuum blood collection $2 \mathrm{ml} / 6 \mathrm{ml}$ & & BD company \\
\hline Slide & & Shitai \\
\hline Cover glass & & Shitai \\
\hline Gun head $200 \mu \mathrm{L} / 1000 \mu \mathrm{L}$ & & Axygen \\
\hline Pasteurella & & NEST \\
\hline Centrifuge tube $15 \mathrm{ml}$ & & Nunc \\
\hline \multicolumn{3}{|c|}{ Instrument } \\
\hline Membrane $8 \mu \mathrm{M}$ & & Wuhan Youzhiyou company \\
\hline CTC biopsy detection equipment D-100 & & Wuhan Youzhiyou company \\
\hline Membrane remover & & Wuhan Youzhiyou company \\
\hline Drying box & & Shanghai Yiheng \\
\hline Single channel pipette $200 \mu \mathrm{L} / 1000 \mu \mathrm{L}$ & & Thermo \\
\hline Microscope Olympus BX41 & & Olympus \\
\hline Tweezers & & Domestic \\
\hline
\end{tabular}

3.7. Immunohistochemical Assay. The biopsies were fixed with $8 \%$ formaldehyde solution for 24 hours, and $8 \mu \mathrm{m}$ thick serial sections were taken for histochemical staining. Ki67 was nuclear stained and yellow: 12 visual fields were randomly observed under $450 \mathrm{X}$-ray microscopes. According to the proportion of positive cells in all the cells, they were divided as positive cells $\leq 15 \%$ were negative and positive cells $>15 \%$ were positive. Over $25 \%$ of the positive cells were highly expressed, and $<25 \%$ were of low expression.

\subsection{Rehabilitation Medical Gymnastics Training. There are} three stages of medical gymnastics training.

The first stage: the patients take the lying position or sitting position, and the nurses guide the patients to exercise the muscle groups of the upper limbs and neck on the operation side, including fist and palm practice, shaking the palm root, hand rubbing, ten rotation, neck flexion, back extension, and body wrapping at the same time, combined with auxiliary massage, through relaxing muscle movement, accelerating lymph and blood return, preventing edema of the affected limb, and alleviating discomfort of the neck, shoulder, and back three to four times a week for 7 weeks.

The second stage: with active and auxiliary movement, the healthy side of the upper limb assists the operating side and shoulder muscles to contract the muscle movement of the upper arm. Through the strength of the neck and body, the fibrous tissue of the patients with axillary scar at the bottom of the collapse is gradually reduced, the shaving situation is alleviated, the shoulder joint activity is gradually improved, and the recovery of the upper limb function of the shoulder joint is slowly promoted three to four times a week for 7 weeks.

The third stage: at-home or outdoor exercise, using cycle and resistance training, muscle groups alternate movement, local and overall combination. Through its forward bending, expansion, abduction, adduction, encirclement, and other full range of motion, the upper arm shoulder joint can strengthen the strength of its shoulder joint-related deltoid muscle, shoulder sub-spleen muscle, supraspinatus muscle, infraspinatus muscle, and other muscle tissue strength, such as dim biceps brachii, obscure triceps brachii, and intercostal muscle three to four times a week for 7 weeks.

The visual analogue scale (VAS), range of motion (ROM) (including flexion, extension and extension), and Barthel index of activities of daily living (ADL) scale were measured and recorded $[14,15]$.

3.9. Evaluation of Clinical Efficacy. RECIST (1.1), revised in 2008, was used by the European Organization for Research and Treatment of Cancer (EORTC), the National Cancer Institute (NCI), and the National Cancer Institute of Canada to assess tumor size and the efficacy of neoadjuvant chemotherapy for breast cancer.

(1) Complete remission (CR): all target lesions disappeared and no new lesions appeared

(2) Partial response (PR): total reduction of baseline maximum diameter of all target lesions $\geq 35 \%$ 
(3) No change (SD): the sum of the longest baseline diameters of all target lesions decreased, PR remained unchanged, and $\mathrm{PD}$ remained unchanged

(4) Progression (PD): the total maximum diameter of all target lesions increased by more than $25 \%$ or new lesions appeared

3.10. Evaluation of the Pathological Effect. In this study, the Fisher score and pathological features of chemotherapy breast cancer edited by the Ministry of Health were used to describe and classify the diagnosis and treatment of breast cancer. After the chemotherapy, the pathological properties of the tumor were degenerative. The cell death was mostly in the piece, interstitial fibrous tissue hyperplasia, and the granulation tissue was infiltrated into the foam cells, lymphocytes, and plasma cells around the nests and nests.

The pathological remission rate was divided into three grades.

(1) Grade I pathological changes (PSD): mild tissue reaction, effective but not sensitive to chemotherapy, and less than one-third of the sections are in the reaction area, infiltrating more cancer components, showing the remaining life of cancer cells and high lymph node metastasis rate

(2) Grade II pathological remission (PPR): mild tissue reaction, mild sensitivity to chemotherapy, chemotherapy is about $1 / 2$ of the response, and the lymph node metastasis of invasive cancer can be seen

(3) Grade III pathological complete remission chemotherapy response (PCR): severe tissue reaction, the effect of chemotherapy, the patient is very sensitive to chemotherapy, and the composition of intraductal cancer tissue section, see infiltration, lymph node and metastasis rate is very low

3.11. Statistical Treatment. SPSS13.0 statistical software was used for analysis. All the measurement data were expressed as mean $\pm \mathrm{SD}$. The indexes of cardiac function before and after chemotherapy were paired/tested. The correlation between the two variables was analyzed by person correlation analysis. Bland Altman analysis was used to determine the consistency boundary between intraobserver and interobserver repeatability.

\section{Experimental Results and Analysis}

4.1. Shoulder Score. At baseline, the scores of the two groups did not conform to the normal distribution, the median and quartile were used to describe, the Mann-Whitney test was used for the control group, and the Wilcoxon test was used for internal comparison because the difference in shoulder score was not normal distribution.

It can be seen from the analysis results in Figure 1 that the shoulder joint score of the two groups at 24 weeks was significantly different from the baseline $(P<0.05)$. The experiment shows that the timely rehabilitation training of gymnastics for breast cancer patients after chemotherapy cannot substantially affect the shoulder joint of patients.

4.2. Patients with UpperLimb Dysfunction. The DASH scores of the two groups at baseline and 24 hours were in accordance with normal distribution. An independent sample $t$ test was used for comparison between groups, and a paired $t$ test was used for comparison between the two groups before and after intervention.

According to the analysis results in Figure 2, there was no statistically significant difference between the two groups in the baseline and control group $(P>0.05)$, but there was a statistically significant difference between the two groups. The DASH score between the two groups at 24 weeks and before and after the intervention group $(P<0.05)$ was determined. The experimental data show that the intervention group through gymnastics rehabilitation training has obvious rehabilitation signs on upper limb function after chemotherapy, which is significantly faster than the control group without gymnastics rehabilitation training. The experimental results further proved that effective gymnastics training for breast cancer patients after chemotherapy is conducive to functional recovery and brings positive effect to the treatment effect.

\subsection{Comparative Analysis of Disease Uncertainty and Coping} Style. In order to evaluate the effect of intervention, the uncertainty of disease and coping style of subjects after intervention were adjusted.

According to the analysis chart in Figure 3, after the intervention, there were statistically significant differences in the overall disease uncertainty, uncertainty dimension, complexity dimension, lack of dimension between information, and unpredictability between the intervention group and the control group $(P<0.05)$, and the score of the intervention group was lower than that of the control group. As a response, the difference in the total reaction score and avoidance response score between the intervention group and the control group was statistically significant $(P<0.05)$. The total score and face response score of the intervention group were higher than those of the control group, while the score of avoidable treatment was lower than that of the control group. The difference in the production response score between the two groups was not statistically significant $(P>0.05)$.

\subsection{Comparative Analysis of Disease Uncertainty and Coping} Style. According to the analysis results in Figure 4, the overall disease uncertainty, uncertainty dimension, complexity dimension, information loss dimension, and unpredictability score of the intervention group before and after intervention were statistically significant $(P<0.05)$, and the scores after intervention were lower than those before intervention. In the aspect of coping, the total coping score and coping score of the intervention group before and after the intervention also had statistical difference $(P<0.05)$, and the score after intervention was higher than that before 


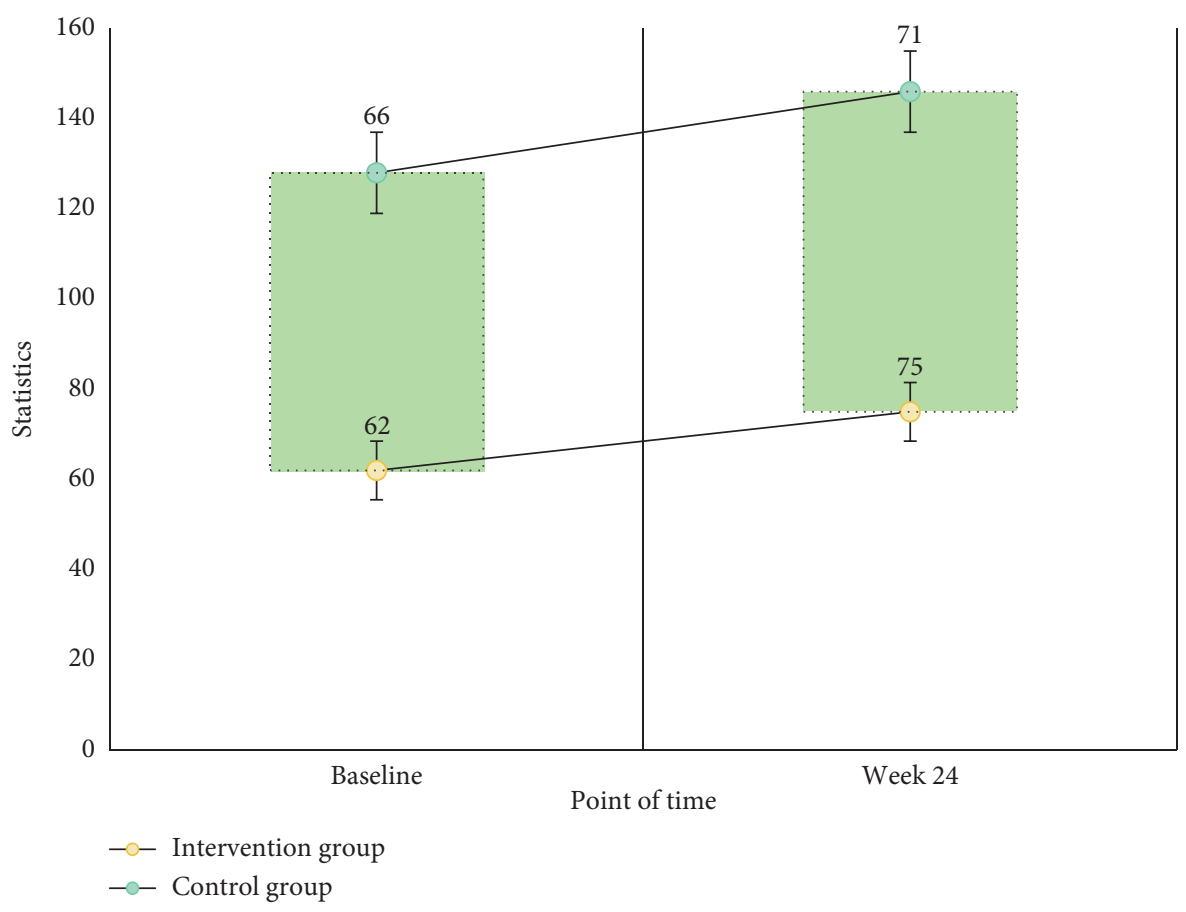

Figure 1: Analysis of the shoulder joint score in two groups.

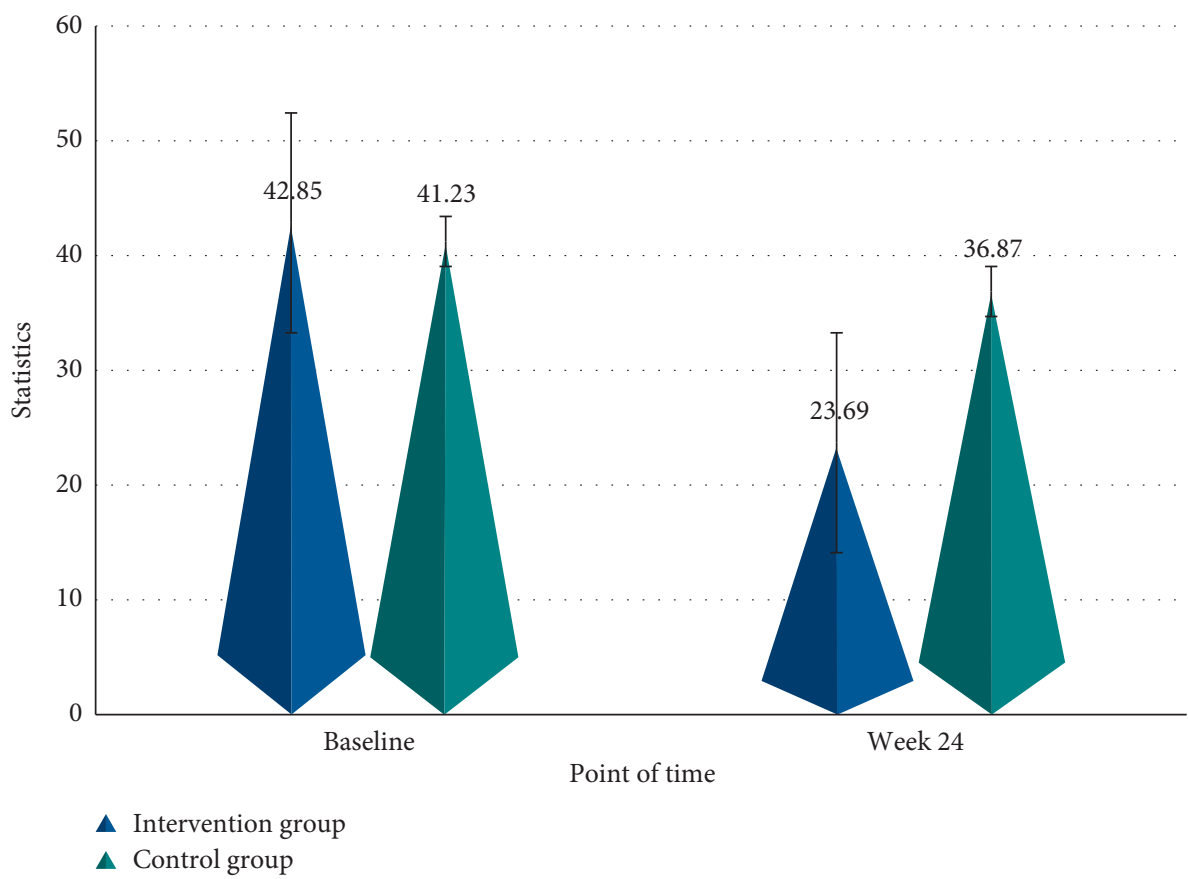

FIgURE 2: Comparative analysis of upper limb dysfunction between the two groups.

intervention, and there was no significant difference in other indexes of the intervention group before and after the intervention $(P>0.05)$. In the control group, there were statistically significant differences in the scores of overall disease uncertainty, uncertainty dimension, and information loss dimension before and after the intervention
$(P<0.05)$. In the aspect of coping, the score of the control group before and after the intervention was statistically significant $(P<0.05)$, and the score after intervention was higher than that before intervention, and there was no significant difference in other indicators of the control group before and after the intervention $(P>0.05)$. 


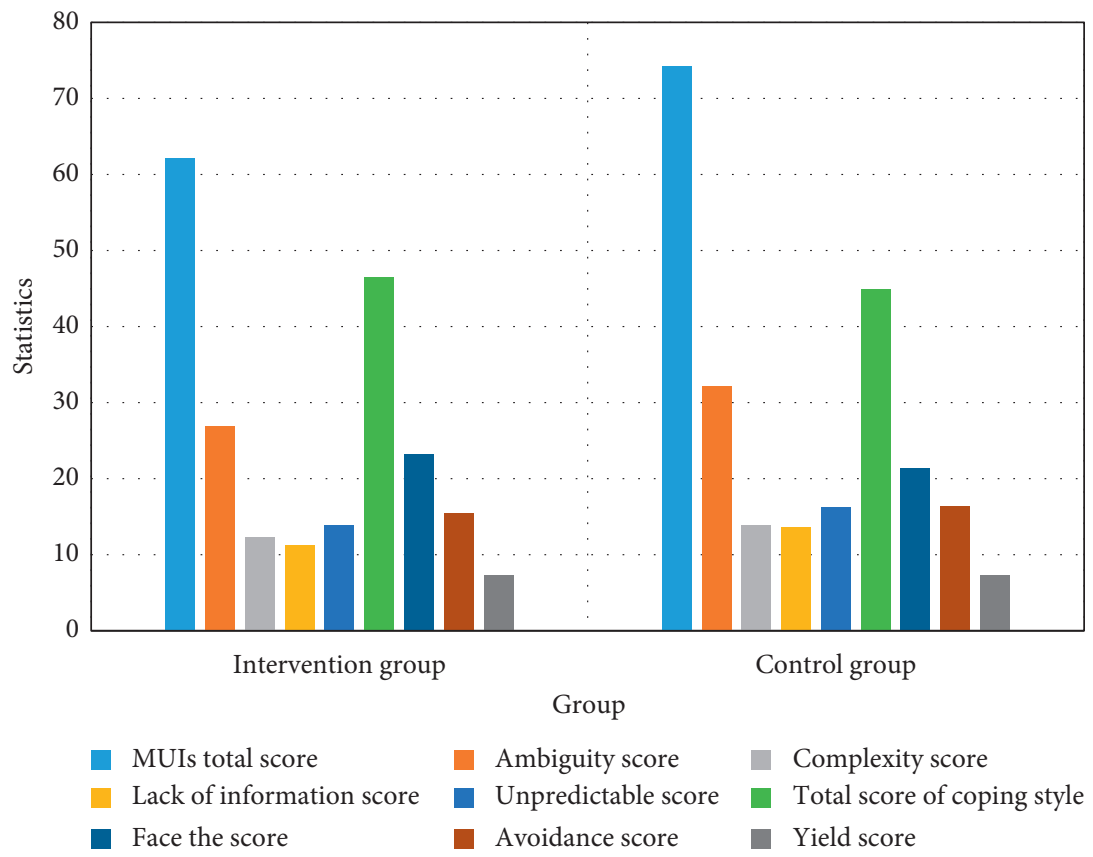

Figure 3: Comparison and analysis of uncertainty of disease and coping style between the two groups after intervention.

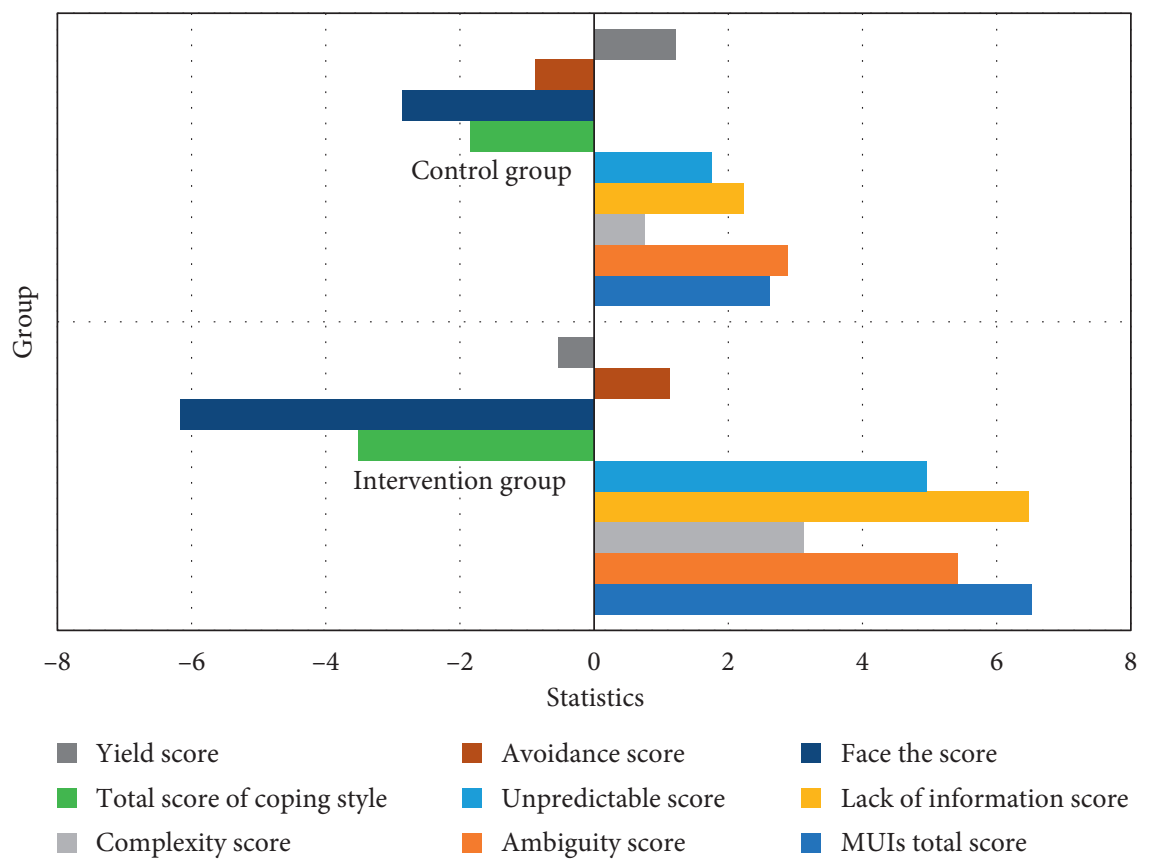

FIGURE 4: Self-comparison and analysis of uncertainty and coping style of patients in two groups before and after intervention.

\section{Conclusions}

The prevalence of breast cancer in China is increasing year by year, which seriously threatens the life and health of women. At present, the pathogenesis of breast cancer is not clear, and genetic factors, environmental factors, behavior habits, and so on will induce breast cancer. At present, chemotherapy and radiotherapy are the main treatment for breast cancer, but no matter which treatment, it will bring serious harm to patients. Although the cure rate of breast cancer has been improved in the recent years, cancer-related fatigue, which is common after operation, is also worthy of attention. Cancer-related fatigue has a serious harm to breast cancer patients. Improving cancer-related fatigue has a positive effect on the treatment of breast cancer. In this paper, through the establishment of gymnastics exercise for breast cancer patients after intervention, we set up a unified evaluation mechanism. The patients were divided into two 
groups: one group was the exercise intervention group, and the other group was the control group. Through a number of comparative experiments including shoulder joint, upper limb dysfunction, and the patient's emotional score, this paper analyzes the experimental data and believes that the current chemotherapy can effectively prevent the spread of cancer cells and improve the cure rate of breast cancer, but the existing postoperative rehabilitation therapy is too backward. The gymnastics rehabilitation therapy in this paper can significantly improve the physical functions of patients after strengthening upper limb training and make the patients' emotions become more positive and sunnier, which is helpful for the postoperative rehabilitation of patients.

\section{Data Availability}

The data used to support the findings of this study can be obtained from the corresponding author upon request.

\section{Conflicts of Interest}

The authors declare that they have no conflicts of interest.

\section{References}

[1] P. De Andrés, S. Cáceres, M. Clemente, M. Pérez-Alenza, J. Illera, and L. Peña, "Profile of steroid receptors and increased aromatase immunoexpression in canine inflammatory mammary cancer as a potential therapeutic target," Reproduction in Domestic Animals, vol. 51, no. 2, pp. 269-275, 2016.

[2] A. Msaki, A. Pastò, M. Curtarello et al., "A hypoxic signature marks tumors formed by disseminated tumor cells in the balbneut mammary cancer model," Oncotarget, vol. 7, no. 22, pp. 33081-33095, 2016.

[3] R. Rostoker, S. Ben-Shmuel, R. Rashed, Z. Shen Orr, and D. Leroith, "Cd24 cell surface expression in mvt1 mammary cancer cells serves as a biomarker for sensitivity to anti-igflr therapy," Breast Cancer Research, vol. 18, no. 1, pp. 1-12, 2016.

[4] J. D. Shull, K. L. Dennison, A. C. Chack, and A. TrenthamDietz, "Rat models of $17 \beta$-estradiol-induced mammary cancer reveal novel insights into breast cancer etiology and prevention," Physiological Genomics, vol. 50, no. 3, pp. 215-234, 2018.

[5] W. Nie, T. Yu, Y. Sang, and X. Gao, "Tumor-promoting effect of il-23 in mammary cancer mediated by infiltration of $\mathrm{m} 2$ macrophages and neutrophils in tumor microenvironment," Biochemical and Biophysical Research Communications, vol. 482, no. 4, pp. 1400-1406, 2017.

[6] J. Cao, M. Huang, Y. Jing et al., "Effect of acupuncture on the expression of er,pr in breast cancer rats induced by dmba," Shanghai Journal of Acupuncture and Moxibustion, vol. 35, no. 3, pp. 344-348, 2016.

[7] Y. Jing, M. T. Bejarano, J. Zaias, and J. R. Merchan, "In vivo anti-metastatic effects of upar retargeted measles virus in syngeneic and xenograft models of mammary cancer," Breast Cancer Research and Treatment, vol. 149, no. 1, pp. 99-108, 2015.

[8] P. Owens, M. W. Pickup, S. V. Novitskiy et al., "Inhibition of bmp signaling suppresses metastasis in mammary cancer," Oncogene, vol. 34, no. 19, pp. 2437-2449, 2015.
[9] E. Ream, G. Gargaro, A. Barsevick, and A. Richardson, "Management of cancer-related fatigue during chemotherapy through telephone motivational interviewing: modeling and randomized exploratory trial," Patient Education and Counseling, vol. 98, no. 2, pp. 199-206, 2015.

[10] N. Stob, M. J. Müller, S. Küpferling, J. D. Schulzke, and K. Norman, "Low recent protein intake predicts cancer-related fatigue and increased mortality in patients with advanced tumor disease undergoing chemotherapy," Nutrition \& Cancer, vol. 67, no. 5, pp. 818-824, 2015.

[11] X. Wang and J. You, "Clinical study of Yiqi Jianpi Recipe in treating lung cancer patients with fatigue associated with chemotherapy," Chinese Journal of Integrated Traditional Chinese and Western Medicine, vol. 35, no. 9, pp. 1069-1073, 2015.

[12] Q. Huang, Z. Yan, and X. Wu, "The effect of concurrent radiotherapy and chemotherapy on cancer-related fatigue and psychological care needs of patients with advanced lung cancer," China Medical Science, no. 1, pp. 117-120, 2016.

[13] D. M. Norden, R. Devine, S. Bicer et al., "Fluoxetine prevents the development of depressive-like behavior in a mouse model of cancer related fatigue," Physiology \& Behavior, vol. 140, no. 3, pp. 230-235, 2015.

[14] A. H. Deyneko, "Formation of culture motor activity of pupils of 5-6 classes by means of basic gymnastics," Pedagogics, Psychology, Medical-Biological Problems of Physical Training and Sports, vol. 19, no. 1, pp. 24-28, 2015.

[15] R. Tanioka, H. Sugimoto, Y. Yasuhara et al., "Characteristics of transactive relationship phenomena among older adults, care workers as intermediaries, and the pepper robot with care prevention gymnastics exercises," The Journal of Medical Investigation, vol. 66, no. 1.2, pp. 46-49, 2019. 